Caracterización fisicoquímica y microbiológica de una bebida fermentada de (Annona cherimola) dulce y seca

Enriquez, M.A

Para citar este artículo: Enriquez, M.A.; (2021). Caracterización fisicoquímica y microbiológica de una bebida fermentada de (annona cherimola) dulce y seca. Pág. 2-12. Santa Fe, Argentina: UNL.DOI 10.14409/fabicib.v24i0.10744 


\section{CARACTERIZACIÓN FISICOQUÍMICA Y MICROBIOLÓGICA DE UNA BEBIDA FERMENTADA DE (ANNONA CHERIMOLA) DULCE Y SECA}

Enriquez, M.A $\mathrm{A}^{1}$

menriquez@uea.edu.ec

1Universidad Estatal Amazónica. Facultad de Ciencias de la Tierra. Campus Central-Puyo-Ecuador.

\section{RESUMEN}

La investigación define las características físico químicas, organolépticas y microbiológicas de una bebida fermentada (dulce y seca) generada a partir de la Annona cherimola, fruta exótica de la región amazónica del Ecuador. La metodología utilizada fue una investigación descriptiva, estableciendo 3 etapas (pre-fermentativa, fermentativa y post-fermentativa). Los análisis físicos químicos llevados a cabo fueron ( $\mathrm{pH}$, densidad, acidez, grado alcohólico) y microbiológicos (coliformes totales y recuento de mesófilos), posteriormente se realizó un análisis estadístico (ADEVA) entre los parámetros físico-químicos de la bebida, resultando significativo; sin embargo se encuentran dentro de los parámetros cifrados como mínimos y máximos en la norma NTE INEN 374. Como conclusión proponemos un proceso fermentativo para esta fruta exótica climatérica de la región amazónica.

Palabras claves: Anona cherimola, vino dulce, vino seco, región amazónica, fermentación, degradación.

\section{SUMMARY}

\section{Physical-chemical and microbiological characterization of a sweet and dry fermented drink of (Annona cherimola).}

The research defines the physical, chemical, organoleptic and microbiological characteristics of a fermented drink (sweet and dry) generated from the (Annona cherimola), an exotic fruit from the Amazon region of Ecuador. The methodology used was a descriptive investigation, establishing 3 stages (prefermentative, fermentative and post-fermentative), the physical chemical analyzes carried out were ( $\mathrm{pH}$, density, acidity, alcoholic degree) and microbiological (total coliforms and mesophilic count ), subsequently a statistical analysis (ADEVA) was carried out between the physical-chemical parameters of 
the beverage, resulting significant, however they are within the parameters coded as minimum and maximum in the NTE INEN 374 standard. In conclusion we propose a fermentation process for this exotic climacteric fruit from the Amazon region.

Keywords: Annona cherimola, sweet wine, dry wine, Amazon region, fermentation, degradation.

\section{INTRODUCCIÓN}

Desde las épocas antiguas, cuando el hombre elaboró por primera vez bebidas alcohólicas por fermentación, se encontró que su ingestión producía una cierta estimulación que ayudaba a alcanzar un nivel de satisfacción, de placer y alegría, pero además de su empleo, estas bebidas a lo largo de los años se han ido perfeccionando con las mejores técnicas, como aquellas que se obtienen a partir de la fermentación de frutas, ricas en azúcares y la utilización de biotecnologías, convirtiéndose en las más consumidas (1). Las frutas son alimentos llamativos por su diversidad de colores, colores y origen, brindan nutrientes y sustancias naturales altamente para la salud (2).

La fermentación es una técnica viable en el desarrollo de nuevos productos con cualidades fisicoquímicas y sensoriales modificadas, especialmente los componentes de sabor y nutricionales. El alcohol y la fermentación del ácido acético y láctico son importantes para la calidad en producción. De estos, la fermentación alcohólica es ampliamente empleada para la preparación de bebidas en las que el alcohol es un constituyente principal (3). Las primeras citas relativas a la tecnología del vino datan de 3.000 años antes de Cristo, incluso antes que la del pan. Sin embargo, el nacimiento de la tecnología industrial enológica data de finales del siglo XIX, cuando Pasteur demostró que las levaduras eran microorganismos vivos causantes de la fermentación y estableció la relación que existe entre la presencia de estos fermentos vivos y la transformación del azúcar en alcohol y gas carbónico (4). A partir de entonces, se han realizado numerosos estudios microbiológicos de aislamiento e identificación de los microorganismos asociadas a la fermentación del vino o bebidas fermentadas. La transformación del jugo de uva en vino es lograda por el desarrollo secuencial de levaduras y bacterias ácido lácticas (5). Si bien son muchos los géneros de levaduras que participan en las primeras etapas, Saccharomyces cerevisiae es la principal responsable de la fermentación alcohólica, es decir, es la levadura que mayoritariamente transforma el azúcar del mosto en alcohol (6). Aunque se encuentra en niveles bajos en la uva, durante la fermentación se multiplican rápidamente, desplazando a los otros microorganismos presentes en el proceso fermentativo.

El aroma y sabor de las bebidas alcohólicas (cerveza, vino, tequila y mezcal) son el resultado de numerosos compuestos volátiles y no volátiles, cuya mezcla compleja define sus atributos sensoriales y la aceptación por el consumidor (7-8-9). La fermentación es una conservación relativamente eficiente y de baja energía, es el proceso que aumenta la vida útil y disminuye la necesidad de refrigeración u otras formas de conservación de alimentos, por tanto, esta tecnología es una técnica muy adecuada para uso en países en desarrollo y áreas remotas donde el acceso a el equipo sofisticado es limitado (10). 
En la NTE INEN 338-1992 (11) se define al vino de la siguiente manera:

- Vino: bebida alcohólica obtenida mediante fermentación completa o parcial de la uva fresca o del mosto de uva (INEN 338, 1992).

- Vino de frutas: bebida alcohólica obtenida mediante fermentación completa o parcial de frutas o del mosto de frutas.

Según Arguello y Luzuriaga (12), el vino es el producto obtenido mediante fermentación alcohólica del mosto de la uva, para lo cual se utilizan equipos especiales para desintegrar la fruta y de la presión para extraer el juego de la fruta para luego añadir agua, azúcar y ácido cítrico. Un buen vino debe presentar las características de sabor de la fruta original, siendo este último el mayor desafío para la elaboración del vino frutal.

Las técnicas más utilizadas según Arozarena (13) difieren en dos factores principales de aquellas para elaborar vinos de uvas, es más difícil extraer el azúcar de la pulpa de algunas frutas que de las uvas y, además, los jugos contenidos de la mayoría de las frutas son más bajos en contenido de azúcar y más altos en acidez que en las uvas.

La Annona cherimola se cultiva comercialmente en España, Chile, Estados Unidos, Australia y Nueva Zelanda. España, que es el primer productor en el mundo: cuenta con algo más de 3.000 hectáreas de plantaciones comerciales de chirimoyo, de las cuales $97 \%$ corresponden al cultivar "Fino de Jete" y el $3 \%$ restante al cultivar "Campas", ambos de origen local (14). Esta es una fruta exótica nativa tropical y subtropical que se ha convertido en un cultivo importante debido a su sabor, alto contenido de pulpa, valor nutricional y uso potencial en medicina popular (15).

La Annona Cherimola es una especie nativa del continente americano y en Ecuador se encuentra adaptada a diferentes condiciones ambientales, desde la provincia de El Oro hasta la provincia de Esmeraldas, desde los 0 hasta los 1000 metros sobre el nivel del mar y a diferentes precipitaciones y humedades relativas. El cultivo se presenta como una de las alternativas más prometedoras para los agricultores ecuatorianos; lamentablemente el desconocimiento de su manejo y necesidades ha provocado que muchas veces sea considerado un producto marginal (16), comúnmente conocida como chirimoya, es una especie de fruta encontrada en diferentes áreas subtropicales alrededor del mundo, principalmente en norte de Perú y sur de Ecuador, cultivada durante los tiempos del Imperio Inca, que datan del 1200 a. de C. La evidencia antropológica sugiere que la fruta era un ingrediente activo en la dieta inca (17).

El objetivo de esta investigación fue definir las características físico químicas, organolépticas y microbiológicas del vino de anona seco y dulce como alternativa de conservación, tomando en cuenta las características de esta fruta amazónica, que se encuentra de forma silvestre. 


\section{MATERIALES Y MÉTODOS}

\section{Material}

Frutos

Las muestras se obtuvieron del cantón Arajuno, ubicado en el cantón Pastaza de la región amazónica del Ecuador, las mismas estuvieron identificadas en relación a su madurez y el contenido de sólidos solubles, se codificaron los tipos de bebida con la siguiente codificación, BS (bebida seca) y BD (bebida húmeda).

estudio se realizó entre los meses de marzo a junio de 2018, con muestras de sangre obtenidas de pacientes de los servicios de UTI, quirófano y guardia del Hospital San Martín de Paraná. Las muestras se obtuvieron, bajo prescripción médica, en el contexto del proceso de atención.

\section{Métodos}

\section{Análisis fisicoquímicos}

Sólidos Totales ( ${ }^{\circ}$ Brix). Para efectuar una medición se agrega al prisma una pequeña cantidad de la muestra homogenizada, utilizando una pipeta, luego se observa y se anota la medición del porcentaje de azúcares.

Potencial de Hidrógeno (pH). Se determinó por potenciometría, se colocó $75 \mathrm{~g}$ de cada una de las tres muestras bien homogenizadas en un vaso de precipitado de $10 \mathrm{~cm}^{3}$ y se leyó directamente con el potenciómetro.

Densidad. Para determinar la densidad de los vinos, se utilizó un picnómetro con termómetro incorporado. Se calculó la densidad del vino de la siguiente manera:

$$
\text { Densidad }=\frac{P . P \text { Vino-P.P vacio }}{\text { P.P Agua }-P . P \text { vacia }} \times \text { Densidad Agua (Ec.1) }
$$

Donde P.P. es el peso del picnómetro lleno con la muestra (agua o vino).

El grado alcohólico se midió con un alcoholímetro cuya escala expresa directamente el contenido de alcohol por lo que no es necesario el uso de tablas.

\section{Análisis microbiológicos}

Coliformes totales. La determinación de microorganismos coliformes totales por el método del Número más Probable (NMP), se fundamenta en la capacidad de este grupo microbiano de fermentar la lactosa con producción de ácido y gas al incubarlos a $35^{\circ} \mathrm{C} \pm 1^{\circ} \mathrm{C}$ durante $48 \mathrm{~h}$., utilizando un medio de cultivo que contenga sales biliares.

Recuento de Mesófilos. La determinación de microorganismos coliformes totales por el método del Número más Probable (NMP).

\section{Análisis Estadístico}

Tiene como propósito proveer información referente a las unidades experimentales que responden a los tratamientos aplicados. El primer paso consiste en someter los datos a un análisis de varianza para establecer si 
hay diferencias significativas entre las medias de los tratamientos. Cuando el investigador efectúa un trabajo experimental para seleccionar el mejor de k tratamientos, esto es, el tratamiento con mayor (o menor) media, o seleccionar un subconjunto, lo más pequeño posible de entre los $\mathrm{k}$ tratamientos, que tenga una alta probabilidad de incluir al mejor o a los mejores tratamientos, no es adecuado que compare todas las parejas posibles de los tratamientos, sino que debe usar un método de selección de medias. En este caso, el análisis de varianza aporta la estimación de la varianza del error con sus grados de libertad y la información básica sobre si existen o no diferencias significativas en las medias de los tratamientos, para el estudio se utiliza Tukey por su simplicidad y por disponer de límites de confianza. Para el estudio se definieron las variables (grados Brix, estado de madurez, y el peso de la materia prima), luego de obtener los resultados, se procesaron usando el paquete estadístico computarizado (versión del software InfoStat 2011).

\section{Proceso Experimental}

En el proceso de elaboración de la bebida se establecieron tres etapas fermentativas:

Fase pre fermentativa. Esta etapa se caracteriza por la extrucción y el reposo de la fruta durante 48 horas, para que se inicie la fermentación, tomando en cuenta las variables de medida (grado Brix, estado de madurez y la masa de la pulpa).

Fase fermentativa. Las pulpas prensadas son llevadas a los tanques de fermentación, donde es regulado el grado Brix y se inocula la levadura. Transcurrido 48 horas se obtiene el mosto.

Fase post fermentativa. La bebida se envasa en frascos de vidrio de $750 \mathrm{ml}$, que poseen características de impermeabilidad, inatacabilidad química y neutralidad con el contenido; el corcho se utiliza como sistema de cierre de botellas, debido a sus cualidades de elasticidad, impenetrabilidad a los líquidos e inalterabilidad, que son indispensables para la conservación del vino. En la tabla 1 se detalla el proceso de obtención de la bebida fermentada.

\begin{tabular}{|c|l|}
\hline $\begin{array}{c}\text { Selección } \\
\begin{array}{c}\text { Preparación del } \\
\text { mosto }\end{array}\end{array}$ & $\begin{array}{l}\text { Obtención de la fruta en buen estado de madurez y que no presente alteraciones físicas y químicas } \\
\text { aerobio óptimo para el desarrollo enzimático. }\end{array}$ \\
\hline $\begin{array}{c}\text { Corrección del } \\
\text { mosto }\end{array}$ & $\begin{array}{l}\text { El mosto se lo diluye en agua y se realiza la corrección de los grados Brix: } \\
\text { Azúcar vino dulce }=24^{\circ} \text { Brix } \\
\text { Azúcar vino seco }=16^{\circ} \text { Brix }\end{array}$ \\
\hline $\begin{array}{c}\text { Activación de la } \\
\text { levadura }\end{array}$ & A una temperatura de $28^{\circ} \mathrm{C}$, dejando en reposo y esperando que se active. \\
\hline $\begin{array}{c}\text { Adición de la } \\
\text { levadura }\end{array}$ & Se le agregará 1 gr por litro. \\
\hline $\begin{array}{c}\text { Titulación del } \\
\text { Mosto }\end{array}$ & Se tomó la medición de los grados Brix y acidez. \\
\hline
\end{tabular}




\begin{tabular}{|c|l|}
\hline $\begin{array}{c}\text { Determinación de } \\
\text { la acidez }\end{array}$ & Se utilizó $5 \mathrm{ml}$ de mosto, $5 \mathrm{ml}$ de agua destilada, 3 gotas de fenolftaleína. \\
\hline Fermentación & $\begin{array}{l}\text { Se utilizó un fermentador con capacidad de } 5 \mathrm{tt} \text {, completamente sellado evitando el ingreso de } \\
\text { oxígeno. }\end{array}$ \\
\hline Pasteurización & $\begin{array}{l}\text { La pasteurización tiene como objetivo frenar totalmente la fermentación este proceso se realizó } \\
\text { en la cocina industrial a una temperatura de } 65^{\circ} \mathrm{C} \text { por } 15 \text { minutos, evitando que el alcohol se } \\
\text { volatilice. }\end{array}$ \\
\hline Embotellado & Se procedió al llenado en botellas de vidrio, para su posterior análisis. \\
\hline Almacenado & Se almacenó en un lugar oscuro y seco. \\
\hline
\end{tabular}

Fuente: elaboración propia.

\section{RESULTADOS Y DISCUSIÓN}

Análisis bromatológico a la bebida fermentada de annona dulce y seca

En la tabla 2 se presentan los resultados del análisis fisicoquímico de la bebida dulce y seca en función de la NTE INEN 0372.

Tabla 2. Resultados Fisicoquímicos

\begin{tabular}{|c|c|c|c|c|c|}
\hline Volumen Final & Tipo & $\mathbf{p H}$ & ${ }^{\mathbf{0}}$ Brix & Densidad & Grado alcohólico \\
\hline 1005 & Dulce & 5.5 & 7.8 & 1.56 & 13 \\
\hline 984 & Dulce & 5.4 & 7.7 & 1.45 & 13.1 \\
\hline 1075 & Dulce & 5.3 & 7.9 & 1.50 & 12 \\
\hline 990 & Seco & 5.2 & 6 & 1.30 & 11.8 \\
\hline 985 & Seco & 4.8 & 6.2 & 1.40 & 10 \\
\hline 1052 & Seco & 4.9 & 6.5 & 1.35 & 9.80 \\
\hline
\end{tabular}

Según Cabrera, Cuenca y Quicazán (18) durante 8 días a $25^{\circ} \mathrm{C}$, determinando pH, acidez titulable (AT) y sólidos solubles ('Brix). Se midió por duplicado acidez volátil (6) y grado alcohólico (NTC 5113) en el producto. Por cromatografía de gases (AOAC.983.13) (Cromatógrafo Agilent 7890, columna Carbowax), se evaluó el perfil de algunos compuestos volátiles, alcoholes superiores y ácido acético. Machado de Castilhos (19) reportó en su estudio de Influencia de dos procedimientos de vinificación diferentes sobre las propiedades fisicoquímicas y sensoriales de vinos tintos, valores promediados de $\mathrm{pH}$ de 3,3 a 3,4; acidez volátil de 0,2 a 0,3; densidad de 0,99 y contenido alcohólico de 9,7 a 12,5. Los valores arrojados en las pruebas fisicoquímicas del aperitivo vínico de lulo son cercanos o se encuentran dentro del rango reportado por Machado de Castilhos (19) y otros autores como Bindon (20), Gómez (21) y Seung-Joo Lee et. al. (22). En la Tabla 3 se presentan los análisis microbiológicos de la bebida seca y dulce. 
Tabla 3. Resultados microbiológicos

\begin{tabular}{|c|c|c|c|c|}
\hline Código & $\begin{array}{c}\text { Volumen } \\
\text { final }\end{array}$ & Tipo & Coliformes totales & Recuento de mesófilos \\
\hline BD1 & 1005 & Dulce & $<200 \mathrm{NMP} / 100 \mathrm{ml}$ & $100 \mathrm{ufc} / \mathrm{ml}$ \\
\hline BD2 & 984 & Dulce & $<100 \mathrm{NMP} / 100 \mathrm{ml}$ & $200 \mathrm{ufc} / \mathrm{ml}$ \\
\hline BD3 & 1075 & Dulce & $<200 \mathrm{NMP} / 100 \mathrm{ml}$ & $150 \mathrm{ufc} / \mathrm{ml}$ \\
\hline BS1 & 990 & Seco & $<300 \mathrm{NMP} / 100 \mathrm{ml}$ & $100 \mathrm{ufc} / \mathrm{ml}$ \\
\hline BS2 & 985 & Seco & $<200 \mathrm{NMP} / 100 \mathrm{ml}$ & $100 \mathrm{ufc} / \mathrm{ml}$ \\
\hline BS3 & 1052 & Seco & $<200 \mathrm{NMP} / 100 \mathrm{ml}$ & $200 \mathrm{ufc} / \mathrm{ml}$ \\
\hline
\end{tabular}

Ufc: Unidades formadoras de colonias, NMP: Método del número más probable, BD: vino dulce, BS: vino seco.

Con los parámetros resultantes hacemos relación entre la NTE INEN 0372 y la NTC 404, 2007, teniendo como resultado que se enmarcan en sus requerimientos. Según Enríquez (23), los métodos de conservación de frutas (bebidas fermentadas) son elementos utilizados por la industria, para minimizar el deterioro causado por diferentes microorganismos, no todos actúan con la misma intensidad frente a mohos, levaduras, y bacterias, de forma que no hay un espectro completo frente a todos los microorganismos.

En relación a los resultados de los análisis fisicoquímicos se presentan los resultados en la tabla 4.

Tabla 4. ADEVA de los resultados fisicoquímicos.

\begin{tabular}{|c|c|c|c|c|c|}
\hline \multicolumn{7}{|c|}{ TRATAMIENTOS } \\
\hline & Vino Dulce & Vino Seco & & & \\
\hline Parámetros & Media & Media & $\mathbf{F}$ & $\mathbf{p}$ & Significancia \\
\hline $\mathrm{pH}$ & 5,40 & 4,97 & 10,56 & 0,0315 & Significativo \\
\hline Brix & 7,80 & 6,23 & 100,41 & 0,0006 & Significativo \\
\hline Densidad & 1,50 & 1,35 & 12,75 & 0,0234 & Significativo \\
\hline Grado alcohólico & 12,7 & 10,53 & 8,89 & 0,0406 & Significativo \\
\hline
\end{tabular}

Fuente: elaboración propia. Nota: $\mathrm{P}<0.05$, Si existe diferencias significativas estadísticas de acuerdo al ADEVA.

Luego de evaluar los resultados estadísticamente mediante un ADEVA, obtenemos una diferencia significativa entre las dos bebidas, en los parámetros establecidos. Por lo que aplicamos una prueba de Duncan, corroborando las diferencias significativas, según se detalla en la tabla 5. 
Tabla 5. Prueba de Duncan

\begin{tabular}{|c|c|c|c|c|c|}
\hline \multirow{2}{*}{ Parámetro } & Tratamiento & Medias & $\mathbf{n}$ & & \\
\hline \multirow{2}{*}{ Ph } & Seco & 4,97 & 3 & $\mathrm{~A}$ & \\
\cline { 2 - 6 } & Dulce & 5,4 & 3 & & $\mathrm{~B}$ \\
\hline \multirow{2}{*}{ Brix } & Seco & 6,23 & 3 & $\mathrm{~A}$ & \\
\cline { 2 - 6 } & Dulce & 7,8 & 3 & & $\mathrm{~B}$ \\
\hline \multirow{2}{*}{\begin{tabular}{c} 
Densidad \\
\multirow{2}{*}{$\begin{array}{c}\text { Grado Alco- } \\
\text { hólico }\end{array}$}
\end{tabular}} & Seco & 1,35 & 3 & $\mathrm{~A}$ & \\
\cline { 2 - 6 } & Dulce & 1,5 & 3 & 3 & $\mathrm{~B}$ \\
\hline
\end{tabular}

Fuente: elaboración propia. Nota: letras distintas indican diferencias significativas $(p<=0,05)$

Pastrana, Durango, De Paula y Acevedo (24) realizaron la caracterización bromatológica, fisicoquímica y microbiológica de Avena Sinuana y de Chicheme, bebida autóctonas de Córdoba, Colombia. Tomaron muestras al azar y los análisis los hicieron por triplicado durante tres días, siguiendo los métodos oficiales de Química Analítica y las Normas Técnicas Colombianas de microbiología. Se realizaron análisis de varianza y comparaciones múltiples $(p \leq 0,05)$. La Avena Sinuana presentó mayores porcentajes de cenizas (1.39), azúcares totales (5.03) y azúcares reductores (5.51). Destacó por el contenido de proteínas (6.77) grasa (1.02) y acidez (0.39). Los recuentos microbianos sobrepasaron los límites establecidos en la Avena Sinuana los más altos fueron mesófilos (3.59) y coliformes totales (3.38). Los resultados revelaron que la bebida tenía una cantidad adecuada de nutrientes, pero elaboradas con deficientes condiciones sanitarias.

Por su parte, Arrazola-Paternina, Barrera-Violeth y Villalba-Cadavid (25) caracterizaron los frutos de la Guanábana cimarrona en tres estados de madurez, a saber, verde, pintona y madura, analizando características físicas y bromatológicas (acidez titulable, pH, ${ }^{\circ}$ Brix, índice de madurez, humedad, cenizas, fibra bruta, contenido de vitamina C, sacarosa mediante Cromatografía Líquida de Alta Resolución, HPLC). Los resultados arrojaron diferencias estadísticas significativas entre los estados de madurez del fruto en sus propiedades bromatológicas y fisicoquímicas, exceptuando el análisis de cenizas como también en sus propiedades físicas. El estudio reportó una composición promedio para el fruto de Guanábana cimarrona así: Acidez titulable 2.6\%; $\mathrm{pH} 4.55 \%$; ${ }^{\circ}$ Brix $11.5 \%$; índice de madurez $0.8 \%$; humedad $81 \%$; cenizas $0.72 \%$; fibra bruta $8.55 \%$; contenido de vitamina C, 3.6\%. Los resultados obtenidos por HPLC muestran una concentración de $1.86 \mathrm{mg}$ kg-1 de Sacarosa, obteniéndose mayor producción en el tercer tratamiento, correspondiente al estado madurez en el cual las características organolépticas y fisicoquímicas son ideales para consumo en fresco o para su procesamiento agroindustrial.

En otro trabajo, Rodríguez-Villacis y Hernández-Monzón (26) desarrollaron una bebida fermentada de lactosuero hipocalórica con la adición de jugo de aloe vera, pulpa de guanábana y cultivos probióticos. Como materias primas se utilizaron suero lácteo dulce, pulpa de guanábana (Annona muricata L), jugo de aloe (aloe 
vera B), edulcorante artificial de la firma Splenda y los cultivos probióticos Bifidobacterium sp., S. thermophilus, L. acidophilus y L. delbrueckii subsp., bulgaricus. Para la elaboración de la bebida láctea el suero se mezcló con pulpa de guanábana y jugo de aloe en las proporciones: pulpa de guanábana 10,15 y 20 \% y el jugo de aloe 5, 10 y $15 \%$, la mezcla se estandarizó al 8 \% de sólidos totales lácteos con leche en polvo descremada y fue inoculada con los cultivos lácticos al $4 \%$ y la fermentación se realizó a $42 \pm 1$ oC. A las formulaciones desarrolladas se les controlaron acidez y aceptabilidad. La mejor formulación de la bebida fermentada hipocalórica fue la de 15 \% de pulpa de guanábana y de 10 \% jugo de aloe, con una calificación de "me gusta". La vida de almacenamiento a 4 oC puede ser hasta de 21 días.

\section{CONCLUSIONES Y RECOMENDACIONES}

De lo discutido en este trabajo es posible plantear algunas conclusiones como:

Existen frutas cuyo índice de acidez se aproxima al de la uva y una vez que el mosto ha sido estandarizado con azúcar, alcanzan las condiciones definidas para obtener una bebida fermentada.

Los tratamientos no aceptados en la bebida dulce y seca corresponden al 33,33 y $40 \%$ en relación a la parte sensorial, lo que nos indica que la fruta en un proceso fermentativo está expuesta a cambios en su olor y color.

Los procesos previos de fermentación que se aplican generan un cambio físico químico y microbiológico, marcados por los análisis de laboratorio y el cumplimiento de las normativas de calidad.

En general podemos concluir que esta especie amazónica climatérica, genera buenos resultados al ser sometida a este método de conservación, lo que genera una oportunidad de transformación de las materias primas a nivel amazónico.

\section{Referencias bibliográficas}

1- Leefers, L.S, 1973. Manual de enología y bebidas alcohólicas 2v. SENA. Servicio Nacional de Aprendizaje. Colombia $146 \mathrm{p}$

2- Enríquez, M., Pérez, M., Montenegro, K., 2019. Formulation and evaluation of a pineapple and strawberry pasteurized pulp mix. Agroindustrial Science, 61-65.

3- Sivasakthivelan, P., Saranraj, P., Sivasakthi, S., 2014. Production of bioethanol by Zymomonas mobilis and Saccharomyces cerevisiae using sunflower head wastes - A comparative study. Int J Microbiol, 208-16.

4- Lafon-Laforcade, S., 1983. Wine and Brandy. En "Bio- En "Biotechnology", vol. 5, Reed, G. Verlag-Chemie. Heidelberg.

5- Ribereau-Gayon, J., Peynaud, E., Sudraud, P. y Ribereau-Gayon, P. 1976. Traite d' oenologie. Sciences er technniques du vin. Tome III. Dunop. Paris. 
6- Dias, D., R. Schwan, E. Freire y R. Serôdio. 2007. Elaboration of a Fruit Wine from Cocoa (Theobroma cacao L.) Pulp, International Journal of Food Science \& Technology, 42(3), 319-329. doi.org/10.1111/j.13652621.2006.01226.x

7- López, M. C. 1999. Tequila Aroma. In: Flavor Chemistry of Ethnic Foods. F. Shahidi, Ch Ho (eds). Kluwer Academic/Plenum Publishers, New York, Pp. 211-217.

8- Vallejo, C. y González C., 1999. Identificación de los compuestos volátiles del tequila. Bebidas Mexicanas 8 , 1-17.

9- Cole, V. C. y Noble, A. C., 2003. Flavor chemistry. In: Fermented Beverage Production (Kluwer Academic/Plenum Publishers), pp. 393-396. New York.

10- Plutowska B, Wardencki W. 2008. Application of gas chromatography olfactometry (GCeO) in analysis and quality assessment of alcoholic beverages-a review. Food Chem 449-63.

11- NTE INEN (Instituto Ecuatoriano de Normalización) 1992. NTE 338. Bebidas alcohólicas, vinos. Quito-Ecuador.

12- Arguello, Y., y Luzuriaga, D.L., 2012. Extracción y aprovechamiento del mucilago de Cacao (Theobroma cacao) como materia prima en la elaboración de vino. Quito UTE.

13- Arozarena, I. 2007. Seminarios Internacionales. Elaboración de vino de frutas. Ambato.

14- Pérez de Oteyza, M.A., 2002. El banco español de germoplasma de chirimoyo. Un poco de historia y situación actual. In: Resúmenes III Congreso Internacional de Anonáceas. Quillota, Chile.

15- Costa E.V., Pinheiro S.M.L., Maia B.H., Duarte M.C.T., Amaral A.C.F., Leon G.M., 2009. Antimicrobial and antileishmanial activity of essential oil from the leaves of Annona foetida (Annonaceae). Quim Nova 32(1):78-81

16- Andrade, D., 2015. Manual del cultivo de la guanábana. El cultivo del guanábano. Obtenido de http://www.ecuaguanabana.com/2015/03/manual-del-cultivo-de-laguanabana.html

17- Bonavia, D., Ochoa, C., Tovar, O. y Palomino, R. (2004). Archaeological evidence of cherimoya (Annona cherimolia mill.) and guanabana (Annona muricata L.) in ancient Peru. Economic Botany, 58, 509-522.

18- Cabrera., S, Cuenca, M y Quicazán, M (2012). Efecto de la proporción de pulpa en el mosto para la producción de bebida alcohólica de guayaba (Psidium guajava). Vitae, 19 (1), S246-S248.

19- Machado de Castilhos, M., 2013. Influence of two different vinification procedures on the physicochemical and sensory properties of Brazilian non-Vitis vinifera red wines, LWT -Food Science and Technology.

20- Bindon, K. 2013. Relationships between harvest time and wine composition in Vitis vinifera L. Cv. Cabernet Sauvignon 1. Grape and wine chemistry. Food Chemistry: 138 (2-3), 1696-1705.

21- Gómez, E. 2011. Volatile and sensory characterization of red wines from cv. Moravia Agria minority grape variety cultivated in La Mancha region over five consecutive vintages. Food Research International: 44 (5), 1549-1560. 
22- Seung-Joo Lee, Jang-Eun Lee, Hyeon-Wee Kim, Sung-Soo Kim, Kyung-Hee Koh, 2006, Development of Korean red wines using Vitis labrusca varieties: instrumental and sensory characterization. Food Chemistry: 94 (3), 385-393

23- Enríquez, M., 2019. Obtención de productos frutícolas deshidratados; tomate de árbol (Cypomandra betacea I) y guayaba (Psidium guajaba I), mediante el empleo de un secador solar con colector plano. Revista Perfiles, 22, vol. 2.

24- Pastrana, Y. I.; Durango, A. M.; De Paula, C. D y Acevedo, D., 2015. Caracterización Fisicoquímica, Bromatológica y Microbiológica de Bebidas Autóctonas de Córdoba, Colombia.

25- Arrazola-Paternina G., Barrera-Violeth, J., Villalba-Cadavid, M., 2013. Determinación física y bromatológica de la guanábana cimarrona (Annona glabra L.) del departamento de Córdoba. ORINOQUIA, Universidad de los Llanos, Villavicencio, Meta, Colombia.

26- Rodríguez-Villacis, D.H.; Hernández-Monzón, A., 2017. Desarrollo de una bebida fermentada de suero con la adición de jugo de Aloe vera y pulpa de fruta Tecnología Química, XXXVII: 46-57. 